\title{
Welfare Technology - You Need to do it Right! A Qualitative Study of the Work of Care Workers to Provide Patient Safety at Home through Technology.
}

\author{
Randi Stokke ( $\nabla$ randi.stokke@ntnu.no) \\ Norwegian University of Science and Technology \\ Aud Obstfelder \\ Norwegian University of Science and Technology \\ Hege Kristin Andreassen \\ Norwegian University of Science and Technology \\ Line Melby \\ Norwegian University of Science and Technology \\ Jørn Isaksen \\ Inland Norway University of Applied Sciences
}

\section{Research Article}

Keywords: Care work, home care practice, safety work, invisible work, social alarm, welfare technology

Posted Date: December 9th, 2020

DOI: https://doi.org/10.21203/rs.3.rs-117272/v1

License: (c) (1) This work is licensed under a Creative Commons Attribution 4.0 International License. Read Full License 


\section{Abstract}

\section{Background}

In health care, the work of keeping the patient safe and reducing the risk of harm is defined as safety work. In our digitised and technology-rich era, safety work usually involves a relationship between people and technologies. Welfare technology is one of the fastest-growing technology-domains in western health care systems. In the marketing of welfare technology, the expectation is that safety is provided simply by being present in patients' homes. Whilst both research and health authorities are concerned with developing cost-benefit analysis and measuring effects, there is a lack of attention to the daily work needed to ensure that technologies deliver patient safety.

This paper aims to describe how patient safety in home care is performed through and with welfare technology. We base our exploration on the social alarm, an established technology that care workers are expected to handle as an integrated part of their ordinary work.

\section{Methods}

We draw on empirical data from three case studies, involving five Norwegian municipalities that use social alarm systems in home care services. Building on a qualitative explorative design, we analyse observations of practice and interviews with the actors involved, in accordance with King's outline of template analysis.

\section{Results}

Our analysis illuminated three co-existing work processes where patient safety is secured: "Aligning people and technologies"; "Being alert and staying calm"; and "Coordinating activities depending on people and technology". Attention to these work processes exposes the detailed practices of safety, and how it is produced in relational practices involving multiple people and technologies.

\section{Conclusions}

We conclude that the three work processes identified are essential if the safety alarm is to function for the end-user's safety. Home-dwelling patients' safety is reliant on the people involved with technologies, and technologies do not create this on their own. The efforts of care workers are a central feature of creating safety in a patient's home, and in doing so, they utilise a repertoire of skills and knowledge.

\section{Background}

\section{Working to maintain patient safety}

The focus on patient safety is a movement within health and care services that arose when the Institute of Medicine in the US published the report To err is human: building a safer health system [1]. Since then, 
although the focus on patient safety has gained increased interest around the world, this is mainly within a hospital setting even though most patients are in primary care, including home care services. There is therefore a need for more knowledge about the safety work performed in home care, especially in light of the care services becoming increasingly complex and thereby involving more risks for the patients [2-4].

To address this gap, in this paper we draw on sociological insights on healthcare work and organisation in particular, in order to explore the work performed to ensure patient safety in care services in depth.

In their pivotal studies, Strauss et al. [5] point to care workers' engagement in different kinds of work activities, and different types are outlined. They stress that organising work is a major part of the total span of healthcare duties. In doing so, they were among the first to challenge the opinion that care work is solely clinical work at the bedside. Other scholars, for example, Davina Allen [6] have followed this line of argumentation, emphasising that 'organising work' is a vital part of care work, and consequently for patient outcome and experiences.

Another work type described is safety work that - not surprisingly - pertains to creating patient safety. This includes not only efforts to protect patients from the safety risks posed by the illnesses but also risks posed by the environment, the relevant technology, the service and the organisation in which the service takes place. In respect of the technology involved, this safety work focuses on both the purchase and installation of the technology and ongoing maintenance of the technology within the context of use. Due to the complexity of the service, there are many unknown risks related to technology in use.

However, other kinds of activities (work types) are also highly relevant to understand care workers' efforts in producing patient safety. One important type of work is machine work, e.g. the practical work with technologies such as syringe pumps, ventilators and blood pressure monitors in addition to digital technologies like telecare solutions and different welfare technologies. Earlier, such work was mostly reserved for care workers in hospitals, but today machine work is becoming widespread also in home care. The dramatically increasing use of technologies has a wide-ranging impact on the delivery and organisation of healthcare, on both professional practice and patients' experience of illness and its management, including the feeling of safety.

Yet another work type is articulation work. This is sometimes popularly referred to as 'work that makes the work work'. In practice, this means all sorts of connection, integration, coordination and organisational efforts conducted so that the patient trajectory evolves in the desired direction [5].

To summarise, home care workers conduct a range of different work types to provide patient safety. These work types are performed simultaneously and intertwined in the daily work of the carers. In addition to the various forms of work performed by healthcare professionals, we should not forget all the work that must be done by patients and next of kin during the course of a caring trajectory [7]. Applying this broad perspective on care work enables us to see the whole range of care workers' activities in the pursuit of creating safety for patients at home, including welfare technology. 
To add to the complexity, care work takes place in complex services filled with 'wicked problems. Wicked problems are complex problems characterised by disagreements regarding the nature of the problem, different and changing stakeholders, competing interests, rules and regulations, part-time workers and other work issues [8]. An example of this is described by T Johannessen, E Ree, I Aase, R Bal and S Wiig [9] in a Norwegian study of safety work in nursing homes and home care. They found that major threats related to patient safety are the heavy workload and the lack of competence among the personnel.

This implies that empirical studies on healthcare work show that such work is hard to plan and largely consists of a way of working where actors need to make adjustments to the practice of the everyday use of technology $[6,10,11]$.

\section{Patient safety through welfare technology}

Policymakers and providers are increasingly suggesting welfare technology as a solution to promote patient safety in patients' homes. We are not the first to point out the shortage of studies on what it takes for welfare technology to promote patient safety in home care [3]. Up to the present, the research in this field has been dominated by effect-studies, an approach that diverts our attention from the effort invested by those involved in the care to make welfare technology work in the first place [6]. Welfare technology is still often promoted as an easy fix to the increasing pressure to deliver high-quality services to ever more patients with ever greater needs $[12,13]$. Previous research on 'technology in use' in other parts of health care shows that gainful implementation of technology in work practices must handle the intricate and rather unpredictable relationships between peoples and technologies $[7,14,15]$.

To explore the efforts of care workers to provide safety through welfare technology, we have focused on the social alarm as a typical example of a welfare technology used to safeguard frail people living independently at home. The social alarm is frequently described as a "plug and play" device, and has been in use in health care services throughout most western countries for 40 years $[11,16]$. Today social alarms are widely adopted in home care services and routinely embedded in service delivery and patients' everyday lives [17]. Thus, the devices can be considered as 'normalised' technologies [18], suggesting that they are easy to use and that the desired effects are easily accomplished [19]. However, there are still issues related to how the alarms are used by the patients, their functions and the organisation of their use within local services providers. For example, the main unit must be carefully installed in the alarm user's home, and the alarm receiver must be able to communicate when the alarm is activated. The patient must know the range of the pendant both inside and outside the house as this varies depending on the building structure and materials. The social alarm is usually organised as an integrated part of the home care services, entailing responsibility for responding to the alarm when activated. Furthermore, as the newer social alarms have the potential to incorporate a range of functionalities, such as fall alarm devices, blood pressure devices and gas and smoke detectors [17, 20,21], the complexity of the interplay between the care workers, patients and the technical specificities of the social alarm, have increased considerably. 
In previous works, the first author has discussed how social alarms paradoxically both provide safety and give rise to a safety risk for the patients [22]. Other research has shown that care workers play an important role in making the social alarm easy to use for the patients $[23,24]$. This illustrates how the 'ease of use' and the 'normalised' social alarm is the outcome of intricate connections between people and technology in everyday care work, resting on the interplay of care workers, patients and the technical specificities of the social alarm.

\section{Technology and care work - two sides of the same coin}

Equally, we may say that care work is the outcome of how technologies and people are intertwined in practical tasks related to the provision of services for dependents. The actors involved in the practices be it technologies or people - emerge as part of a web of relationships and mutual dependencies to which they contribute [25]. Thus, the technologies are not 'things' with a specific feature that have particular effects when implemented correctly, but specific practices that are inherently contingent, unfolding in specific places, time and contexts. Care work is not just based on 'pure' human relationships, but shaped by continuous interconnections of devices, tools, routines, symbols, knowledge, rules and people, which must continuously be maintained $[26,27]$. The smallest changes in the technologies have the potential to impact on the intricate network of people and technologies that care work is made up of.

In this paper, we highlight how these complex relations between welfare technology and care workers' activities play out in daily practice by asking what the care workers do to make welfare technology function as a tool for patient safety. Our research is based on an empirical study in five Norwegian municipalities where we employ theoretical perspectives of technology in use. By doing so, we aim to provide insight into what this safety work implies and how it is performed in the day-to-day care work.

\section{Methods}

This paper aims to describe and analyse the complex relations between welfare technology and safety work in home care services. We wished to pursue our ethnographic curiosity and contribute knowledge about the details of daily care work in technology-supported home care services by exploring how care workers engage and work with and around the social alarm in order to achieve safety and independent living for their patients.

\section{Methods for data collection}

The empirical material was originally collected in three separate projects involving five Norwegian municipalities that all investigated the implementation of social alarms (see Table 1 [24, 28-30]. The material was joined in one corpus of data for this study. All projects had qualitative, explorative designs. Data were collected in a combination of participant observation, individual interviews and focus group 
interviews following a qualitative study design. This gave the researcher insights into how the care workers ensured patient safety when working with the social alarm

In all five municipalities, social alarm systems for home-dwelling patients had already been implemented prior to our research. The systems were all administered by care workers, mainly registered nurses.

Table 1

The three cases included in the study

\begin{tabular}{|llll|}
\hline $\begin{array}{l}\text { The } \\
\text { three } \\
\text { cases }\end{array}$ & Methods & Characteristics of interview participants \\
\hline Case 1 & $\begin{array}{l}\text { Two } \\
\text { municipalities }\end{array}$ & $\begin{array}{l}\text { Individual interviews } \\
\text { 2-week participant } \\
\text { observations }\end{array}$ & $\begin{array}{l}\text { 6 care workers, two of whom are key } \\
\text { workers responsible for the social alarm }\end{array}$ \\
\hline Case 2 & $\begin{array}{l}\text { Two } \\
\text { municipalities }\end{array}$ & Focus group interviews. & 17 care workers and 1 technician. \\
\hline Case 3 & $\begin{array}{l}\text { One } \\
\text { municipality }\end{array}$ & $\begin{array}{l}\text { Focus group interviews } \\
\text { and individual interviews }\end{array}$ & $\begin{array}{l}12 \text { care workers and } 2 \text { management } \\
\text { workers }\end{array}$ \\
\hline
\end{tabular}

\section{Analytical approach}

The analytical procedure followed an inductive-deductive approach. We built on King's outline of template analysis [31]. Template analysis is a practically oriented, iterative thematic approach that approaches the topic from differing perspectives and rich descriptions, and can be used within different research traditions [32].

Template analysis involves the following steps:

First, an initial template, based on one transcript from each case, was developed. From this, a coding scheme that set out four a priori (tentative) themes were created. These were defined through the initial analysis of the three cases as presented in Table 2. 
Table 2

Tentative advanced themes

\section{Tentative themes:}

Articulation of the alarm work. How is the alarm work articulated by the care worker?

Alarm work as additional work.

When welfare technology functionalities collide with established routines.

Emotions and sense-making - Expectations, pressure, uncertainty and other aspects of work.

The coding scheme opened up for an iterative process for further analysis of the whole empirical material, allowing for "modifying and re-applying the initial template" [31] through an exploration of the data and discussions in the research group. This allowed for a hierarchical organisation of codes, where groups of similar codes were clustered together to produce more general, higher-order codes, and the final template was developed. Table 3 is an audit trail describing how the template was developed by describing the steps carried out through the analysis.

Table 3

The audit trail presenting the analysis

\begin{tabular}{|c|c|}
\hline & Steps of the audit trail \\
\hline 1 & Developing 4 priori themes for the templates as presented in Table 2. \\
\hline 2 & Developing an initial template through analysing one transcript from each case (Table 2). \\
\hline 3 & Developing themes and codes. \\
\hline 4 & Coding data from the full set of transcripts \\
\hline 6 & Distribution of codes within and across transcripts \\
\hline 7 & Providing a synthesis of data from the transcripts within the different codes \\
\hline 8 & Identifying main categories from the codes \\
\hline 9 & Identifying and discussing relationships between themes. \\
\hline 10 & Identifying quotes to illustrate the results \\
\hline
\end{tabular}

\section{Results}

By describing how they worked with the social alarm to ensure safety for the patients, the home care workers created a chronological story of the technology in use, describing the journey of integrating the technology in the care practice. 
The analysis illuminated three main categories (see Table 3: step 8) of work that care workers perform to enable the social alarm to contribute to safety and independent living for the users: "Aligning people and technologies", "Being alert and staying calm" and "Coordinating activities depending on people and technology". Below we describe the detailed work practices involved in the three processes.

\section{Aligning people and technologies}

In all the municipalities included in the study, the patient applied to the municipality for the social alarm when in need of the service. The municipal care workers then performed a formal assessment of whether the social alarm was a suitable service. Aligning users and alarms can be considered the first step in creating safety for people who live at home:

Selecting patients is thus not a straightforward process but triggered thoughts and reflections on which patients should have a social alarm and what kind of alarm they should have.

Providing patients with the right kind of devices and hence provide the right kind of care and safety was not straightforward and could be demanding in terms of expertise and time. The choice of the right technologies was characterised by negotiations between healthcare workers, patients and next of kin. It was necessary to align their wishes, ability to cope and needs with technologies aiming for patient safety. Additionally, the care workers also needed experience with and knowledge of relevant technology. This could be a burden for the care workers, as we can see from the next quote:

"We cannot familiarise ourselves with everything. Which alarm is most suited for that patient? We know what they need, but it's not easy for us to find the right type. (...) And maybe you also have to try out different types. And you have to monitor what happens. There are constantly new technologies being introduced, so following the development... [is time-consuming]."

To avoid a mismatch between a patient and the technology, the care workers needed to draw on several aspects of their professional competence and carry out different types of tasks.

\section{Setting up the alarm: getting it to function.}

The next step was to set up the alarm in the patient's home. This comprised adjusting the particular alarm to the patient's environment and the homecare services' work routines. Informants described the work of setting up the social alarm at the patient's home as comprehensive. Furthermore, after setting it up, they had to ensure that the social alarm was always functioning. The extract below illustrates the wide range of tasks involved in this work-process:

I have the responsibility for all decisions on new social alarms. That means I must find a suitable alarm, send a form to the alarm centre, and install the alarm. Sometimes I try to delegate the task. I have tried to show others how to do it (... ) I buy a SIM card and fix it. We have decided that the municipality will take 
care of this. If it was left to the individual patient, it would delay the process and it would be less transparent (...). And if there are errors in relation to the alarms, I will always be kept informed - they just write messages to me describing the error. But there are over a hundred alarms now, and there are errors regularly. So now I have said [to the patient] that you have to bring the alarm to the alarm centre Monday or Thursday or call them on the telephone. Because I cannot repair an alarm. (...) And it's my job to deactivate social alarms. If somebody dies or moves, I will send in a form and the alarm will be deactivated. I might also go to their home and fetch the alarm, or I will call their next of kin and ask them to deliver it to us.

The care worker's story illustrates the wide range of tasks needed to get the social alarm functioning, and thereby produce patient safety. Patient safety is achieved through combining procedures, administrative skills, and collaboration with home care recipients and their next of kin. Collaboration between the home care services and their partners is also crucial to the alarm system infrastructure. Our analysis revealed that there were requirements for the delivery and organisation of safety work/patient safety that involved multiple actors that needed to be aligned. Some collaborated with technology vendors; others with another department within their municipality, or both. The technical service department or fire department were often involved because they have round-the-clock services and technical staff. An informant with special responsibility for the social alarm described how difficult it was to collaborate with others regarding this due to different work hours. Moreover, it was often unclear whose responsibility it was when something went wrong. Even though the technical work with the alarm was straightforward, sometimes things became challenging and her technical skills fell short:

..I had many conversations with him (the technician) when I went out to connect an alarm. I called him and said: 'I am standing here: where should I put the wires?' Yes, and then he remembered exactly what the alarm looks like underneath, what connections there are... and what the different contacts looked like, so he explained the process to me: First you put the white wire in the middle hole of the alarm device... And that is where I got guidance.

The social alarm practice was dependent on practical negotiations and management involvement. These practices are complex in the sense that home care workers must draw on different kinds of knowledge and a wide range of other people as well as practical skills in order to complete tasks. Furthermore, these tasks require time and attention and they are added on top of the care professionals' traditional 'care tasks.

\section{Responsibility for the alarm: "Being alert and staying calm"}

When a user pressed the alarm, a signal was transferred to a mobile phone. On each shift, individual care workers were given the responsibility for the 'alarm phone'. The care workers responsible for managing the alarms on a shift were not exempted from other care tasks, and they handled the 'alarm phones' in addition to these. 
The care workers described how the alarm calls had priority over other forms of care work as the alarm calls often involved possible life-threatening situations:

.. then we must go there at once because the person might have fallen and injured themselves.. or.. they might even die...

Even though many of the informants described how the social alarm was always prioritised, one care worker described how she sometimes ignored the alarm for a short time when busy with another patient. She further describes how this felt wrong. Whatever choice the care workers made regarding priorities in these situations, it had direct consequences for patient safety, both for the patient who had triggered the alarm and for the patient who was left behind.

The care workers described hectic workdays where they had lists of patients they had to attend to and help in their home. Work in connection with the social alarm normally came on top of everything else. The care worker was usually involved with another patient when the alarm was activated, interrupting the interaction and care since they left the patient in order to answer the alarm call due to client confidentiality. This was a nuisance for both the personnel and the patient, as they spent a lot of time answering the alarm phone. The person receiving care in their home could be in a vulnerable situation, perhaps with parts of their body exposed for wound cleaning, bathing or other care procedures. Care workers thus had to prioritise between giving attention to different patients and their safety. Sometimes the patients whom the care worker was visiting at the time of the alarm commented on these interruptions. However, in general they sympathised with the demands imposed on the care worker.

Some (talking about the patient) are very understanding. They're used to us going in and out of situations. They feel sorry for us when we have to leave the room all the time.

The care workers never knew if, when and how often the alarm would be activated during a shift, and they never knew what they would be exposed to during this work, or how time-consuming it would be. This kept them constantly alert and prepared for dramatic events. They explained that this led to stress, as they had to answer the alarm and be with another patient at the same time. The informants experienced that others did not understand the work and responsibility of having the alarm phone.

Informant: I don't think people are aware of what it involves for us, maybe. We do have a great responsibility. We do have (the alarm) all the time when we are at work.

Informant: You never know what will happen.

In two of the cases included, patients had mobile social alarms, allowing them to use the alarm away from the home. This opened for new freedom for the patients, but also further extended care workers' responsibility for the patients' safety.

Informant: Is it so that they can take it (the alarm) with them to the shop? Are we supposed to come if the alarm is activated? That is a tremendous responsibility for us.

Page 10/18 
The mobile alarm enabled users to travel to their holiday home or elsewhere, and still be able to activate the alarm. This forced the service to think ahead and consider what services to offer the service users. The service is extended so the patient can stay more active, but care workers' responsibilities increased even more. It might be difficult to find the patient when the alarm was activated.

Informant: And everybody says: So, you have a social alarm, that's nice. But they don't know what it implies. They know it provides safety for our patients, but they don't know what lies behind it in relation to the emotions, feeling of responsibility, bad conscience etc. for those in the service.

Being responsible for the social alarm entails professional assessment of both the needs related to the alarm and insights into what the activation of an alarm implies. Furthermore, the effectiveness of the alarms as technological products depends on their integration into other organisational arrangements so as to provide an around-the-clock service. This work is further described below.

\section{Coordinating activities depending on people and technology}

To succeed with social alarms, care workers apparently had to continually adjust procedures, and were involved in negotiations, giving guidance, tinkering and working out procedures. In short, they had to put in place a structure that the patients could utilise when in need.

Technological failure caused some of the informants to wonder whether they could rely on the technology. They described how patients sometimes worried about whether the alarm worked, especially when they struggled to make it function.

"Daring to rely on technology. Daring to rely on sensors, handling the reception of alarms. Here ... there are quite a few changes."

Informants also described how they worried about the patients and were afraid that the technology would not work when needed, particularly when mobile phone signals in the area were unstable. This made them worry about whether to trust the system:

It was stressful, never knowing whether the alarm system worked. When you discovered that the system was malfunctioning, you wondered: Who is lying out there now. Who has activated the alarm?

Competencies, or the lack of competencies, was evident as interviews were probed with different descriptions of how the care workers needed know-how knowledge, not just knowing what do and when to do it, but also what to do when needed. These competencies require special training and education, depending on the context in which the competencies will be used.

"...it means a lot of practical work for the health worker when a user is assigned an alarm. It requires expertise in many areas: Technical, organisational, communication, cooperation, etc. 
Another practice born out of the technologies was the delegation of responsibility towards the care workers, which the previous quote also touches upon. This is explicit in the following citation where the care worker describes changes when they implemented new alarms:

Informant: "[the new system] increases the users' feeling of being kept safe when they know that they hear us loud and clear and that we respond quickly. It also increases the feeling of security for the care workers when several of us are logged on to [the alarm system]. This functionality allows us to tell the system that we are busy, and then the alarm will be delegated to the next care worker. On the old system we had two alarm telephones and [on this system we could not report as busy]. Now [with the new system] we hear them when they [the users] press the alarm. Often, they just want to ask a question. Previously we would have made a visit to determine the cause of the alarm. [...] There's a sense of security in the fact that you know, if you are busy, the alarm will go to another care worker, or it will return to you."

This quote underlines how the new system brings a sense of safety to both user and care worker, through the functionality of responsibility delegation. We can extrapolate from the quote that care workers' responsibility is increasing, but at the same time, they can resolve situations faster. By not being constantly interrupted by alarm calls, the care workers can (in theory at least) work more efficiently on their current task.

Most of the informants' reported challenges were linked to establishing good practices around the use of social alarms. One municipality reported successfully using a written record of the practice and focusing on establishing routines for information flow. The written record was perceived as crucial to ensuring consistent practice across employees in their organisation. This resonates with other findings describing how training and information flow created employee confidence. Lack of training and information flow caused a failure to cope, especially for new employees and temporary staff:

"It's very important that we are trained so we all feel safe (about the alarms). What l've experienced many times with new employees or temporary staff, is that they say they have the necessary knowledge and skills, but in reality, they do not master the necessary skills"

In sum, the informants described safe social alarm practice as depending on factors such as the number of care workers available; whether they are visiting a patient; the geography of the municipality; and where the care worker is at the time of the alarm activation.

\section{Discussion}

Our analysis has shown that installing, using and maintaining the social alarm in home care services are basic elements of safety work, and hence safety for home-dwelling patients. Ensuring that the alarms were functioning turned out to be a major part of home care staff's work. The seemingly trivial work of making the alarm work, was at the same time stressful for staff because the consequences of a nonfunctioning alarm could be fatal. 
The analysis has also shown that three work processes need extra attention from care workers in order to successfully deliver health services that ensure independence and patient safety through using a social alarm. These are: enrolling 'suitable' users and technologies as well as matching them, the balance between staying alert and keeping calm, and the coordination of activities that depend on people and technology. We argue that attention to these work processes/elements must be considered a requirement for the delivery and organisation of safety work through and with welfare technology. However, whilst care workers in daily practice already attend to them, the tasks involved often remain invisible to managers, health technology vendors and policymakers.

This study has shown how technology forces new roles and new practices on the care workers to provide patient safety using welfare technology. We also see one of the most crucial aspects of the "technology will replace people workers" debate - someone must assume responsibility and act when the technology triggers the alarm. Moreover, a technology supervisor is needed to sort out bad data from good data. In the case of the social alarm, this means distinguishing between false alarms and real alarms, which could mean the difference between life and death.

In addition to the various forms of work performed by the care workers, one should not forget all the work that must be done by patients and next of kin during a caring trajectory. The homecare patients actively participate in shaping the use of the social alarm. The patient receiving care services is inevitably a part of the service, co-creating care with other actors involved. Care workers can offer a service, but it is in the interaction between the care provider and the patient that the care takes place [33]. Furthermore, the integration of technologies tends to result in the delegation of responsibility and work to care workers, the patient and the next of kin [34]. An example of this in the case of the social alarm is how the users must wear the alarm pendant and activate the alarm when necessary.

Care workers' ability to identify a practical need for assistance and attendance to promote patient safety is important. The same applies to knowing when it is appropriate for technology to replace practical assistance. It is important to be observant of what features the technology should supply to the patient.

From this analysis, we conclude that the idea of technology as an "easy fix" to challenges and risks posed when patients live at home must be subject to scrutiny. We have revealed how care workers, patients and next of kin are aware of the complexity of the work to make the social alarm work.

It is important to make nuanced assessments of goals, intentions and the consequences of using the technology in question. Technologies that are perceived as easy to understand and use and that have low complexity will be more likely to be accepted and disseminated (Greenhalgh, Robert, Macfarlane, Bate, \& Kyriakidou, 2004).

There are many indications that technology competence should be more clearly included in the health and care services and professional health educations. Implementation of welfare technology for patients is linked to knowledge of the individual's preferences and needs, and must be linked to these (Demiris et al., 2008). In the development of technical specifications and training, one must ensure that the

Page 13/18 
technology is adapted to the users' requirements, with a range of opportunities for individual adaptation (Shapira et al., 2007).

\section{Conclusions}

This paper has explored how patient safety in home care is performed through and with welfare technology. Based on qualitative data from Norway on the use of social alarms that are widely adopted in home care, we identified three co-existing work processes that are required for the safety alarm to work as intended in providing safety for the patients. Home-dwelling patients' safety is not created by the social alarms on their own but is produced and reproduced through the efforts of care workers vis-à-vis the patient and the technology. Patient safety is the outcome of how the care workers relate to the devices and to the additional workload entailed in ensuring that these devices function optimally for their patients, including appropriate reactions to alarm signals.

Consequently, we argue that safety work is a particular type of care work that overlaps with other types of work such as comfort work, bodywork, organising work and machine work. Safety work, like the other work types, is seldom articulated by those involved, yet it requires attention, skills and knowledge to perform. Furthermore, we claim that the social alarm cannot function on its own, and its implementation can never be finalised. Rather, the social alarm is, and always will be, a continuous everyday process requiring the interaction of care workers, patients, vendors, managers and policymakers in sensible ways. Finally, we believe that there is a need to raise awareness of how workers in home care constantly align people and technologies, focus on being alert and staying calm at the same time, and also coordinate activities related to making the social alarm work. This should also be dealt with on a practical level.

To ease the pressure on care workers in an increasingly technology-intensive work context, and to ensure that patient safety is achieved as smoothly as possible when a growing number of welfare technologies are implemented in home care, some aspects need further clarification. Topics that should be discussed include the division of labour on home care shifts, the need for new routines and education in welfare technology for care workers, and not least: how, and on what level, should decisions on welfare technology implementation and organisation be made. The alarm management practices include procedures, administrative skills, collaboration with home care recipients and their next of kin, and collaboration between the home care services and their partners, which is crucial to the alarm system infrastructure.

\section{Declarations}

\section{Ethical approval and consent to participate}

Norwegian Centre for research data (NSD) granted approval for the project (project numbers 38605,48625 and 43151). Ethical approval in line with the Norwegian Health Research Act was not applicable. All methods were carried out in accordance with relevant guidelines and regulations. The 
municipal health care management in each municipality gave consent to the collection of data within the home care services. All respondents received written and verbal information about the study and were informed that they could decline to be observed or interviewed. All participants gave informed consent to interviews and observations.

\section{Consent for publication}

Not applicable.

\section{Availability of data and material}

The empirical data for the current study consisting of interviews and field notes are not publicly available for privacy reasons. The data is containing information that could compromise research participant privacy/consent. Consent to share anonymised interview transcripts was not asked for at the time of the research. Requests regarding the data from this study might be directed to corresponding author Randi Stokke.

\section{Competing interests}

The authors declare that they have no competing interests.

\section{Funding}

The corresponding author was a doctoral candidate employed at NTNU Norwegian University of Science and Technology. Additionally, the data collection was partly funded by the Regional Research Funds (RFFInnlandet) and partly by the Norwegian directorate of health. The funding body did not have any role or make any decisions concerning this study or the decision to submit the paper for publication. The researchers were independent of the funder.

\section{Authors' contributions}

RS coordinated the work, but all authors have made significant contributions to the manuscript. All authors contributed to the design of the study. RN, JI, LM and AO conducted the data collection. All authors participated in the analysis process. The manuscript was drafted in close collaboration between $\mathrm{RN}, \mathrm{LM}, \mathrm{HKA}$ and $\mathrm{AO}$ with input from $\mathrm{Jl}$. All authors read and approved the final manuscript.

\section{Acknowledgements}


Thanks to researchers from NTNU in Gjøvik who participated in part of the data collection and Yrjan Kvam for participating in some of the early analysis.

\section{References}

1. Kohn LT, Corrigan JM, Donaldson MS, McKay T, Pike K: To err is human. building a safer health system 2000, 600:2000.

2. Wilson T, Pringle M, Sheikh A: Promoting patient safety in primary care: research, action, and leadership are required. In.: British Medical Journal Publishing Group; 2001.

3. Guise V, Anderson J, Wiig S: Patient safety risks associated with telecare: a systematic review and narrative synthesis of the literature. Bmc Health Serv Res 2014, 14(1):588.

4. Lawati MHA, Dennis S, Short SD, Abdulhadi NN: Patient safety and safety culture in primary health care: a systematic review. BMC family practice 2018, 19(1):104.

5. Strauss AL, Fagerhaugh SY, Suczek B, Carolyn W: Social organization of medical work. New Brunswick, N.J: Transaction Publishers; 1997.

6. Allen D: The invisible work of nurses: Hospitals, organisation and healthcare. Abingdon: Rooutledge; 2014.

7. Pols J: Good relations with technology: Empirical ethics and aesthetics in care. Nursing Philosophy 2017, 18(1).

8. Roberts N: Wicked problems and network approaches to resolution. International public management review 2000, 1(1):1-19.

9. Johannessen T, Ree E, Aase I, Bal R, Wiig S: Exploring challenges in quality and safety work in nursing homes and home care-a case study as basis for theory development. Bmc Health Serv Res 2020, 20:1-12.

10. Star SL, Strauss A: Layers of silence, arenas of voice: The ecology of visible and invisible work. Computer supported cooperative work (CSCW) 1999, 8(1):9-30.

11. Procter R, Wherton J, Greenhalgh T, Sugarhood P, Rouncefield M, Hinder S: Telecare call centre work and ageing in place. Computer Supported Cooperative Work (CSCW) 2016, 25(1):79-105.

12. Moser I, Thygesen $\mathrm{H}$ : Exploring possibilities in telecare for ageing societies. In: Ethics of care: critical advances in international perspective. edn. Edited by Barnes M. Bristol: Policy Press; 2015: 111-123.

13. Greenhalgh T, Procter R, Wherton J, Sugarhood P, Hinder S, Rouncefield M: What is quality in assisted living technology? The ARCHIE framework for effective telehealth and telecare services. $B M C$ Medicine 2015, 13(1):1-15.

14. Greenhalgh T, Procter R, Wherton J, Sugarhood P, Shaw S: The organising vision for telehealth and telecare: discourse analysis. BMJ open 2012, 2(4).

15. Johannessen TB, Storm M, Holm AL: Safety for older adults using telecare: Perceptions of homecare professionals. Nursing open 2019, 6(3):1254-1261. 
16. Heinbüchner B, Hautzinger M, Becker C, Pfeiffer K: Satisfaction and use of personal emergency response systems. Zeitschrift für Gerontologie und Geriatrie 2010, 43(4):219-223.

17. Fisk MJ: Social alarms to telecare: Older peoples services in transition. Bristol: Policy Press; 2003.

18. May C, Finch T: Implementing, Embedding, and Integrating Practices: An Outline of Normalization Process Theory. Sociology-the Journal of the British Sociological Association 2009, 43(3):535-554.

19. Pols J: Telecare What patients care about. In: Care in Practice. edn. Edited by Mol A, Moser I, Pols J. Bielefeld: Transcript Verlag; 2010: 171-194.

20. Sugarhood P, Wherton J, Procter R, Hinder S, Greenhalgh T: Technology as system innovation: A key informant interview study of the application of the diffusion of innovation model to telecare. Disability and Rehabilitation: Assistive Technology 2013, 9(1):79-87.

21. Pritchard GW, Brittain K: Alarm pendants and the technological shaping of older people's care: Between (intentional) help and (irrational) nuisance. Technological Forecasting and Social Change 2015, 93:124-132.

22. Stokke R: The Personal Emergency Response System as a Technology Innovation in Primary Health Care Services: An Integrative Review. Journal of Medical Internet Research 2016, 18(7):e187.

23. Sánchez-Criado T, López D, Roberts $C$, Domènech M: Installing telecare, installing users: Felicity conditions for the instauration of usership. Sci Technol Hum Val 2014, 39(5):0162243913517011.

24. Stokke R: Older People Negotiating Independence and Safety in Everyday Life Using Technology: Qualitative Study. Journal of medical Internet research 2018, 20(10).

25. Nicolini D: Practice theory, work, and organization: An introduction: OUP Oxford; 2012.

26. Heath C, Luff P, Svensson MS: Technology and medical practice. Sociology of Health \& illness 2003, 25(3):75-96.

27. Sandelowski M: Devices \& desires: Gender, technology, and American nursing: UNC Press Books; 2000.

28. Batt-Rawden KB, Björk E, Waaler D: Human factors in implementation and adoption of innovations in health care services: A longitudinal case study on the introduction of new technology. The Innovation Journal 2017, 22(3):1-25.

29. Stokke R: "Maybe we should talk about it anyway": A qualitative study of understanding expectations and use of an established technology innovation in caring practices. Bmc Health Serv Res 2017, 17(1):657.

30. Isaksen J, Paulsen K, Stokke R, Skarli J, Melby L: Hvilken nytte har hjemmeboende med hjelpebehov av velferdsteknologi? Tidsskrift for omsorgsforskning 2017.

31. King N: Doing template analysis. Qualitative organizational research: Core methods and current challenges 2012, 426.

32. King N: Doing template analysis. Qualitative organizational research: Core methods and current challenges 2012, 426:77-101. 
33. Osborne SP, Radnor Z, Strokosch K: Co-Production and the Co-Creation of Value in Public Services: A suitable case for treatment? Public Management Review 2016, 18(5):639-653.

34. Oudshoorn N: Telecare technologies and the transformation of healthcare. Houndmills, Basingstoke: Palgrave Macmillan; 2011. 